\title{
Vorwort zur deutschen Ausgabe
}

\author{
Von der Feindschaft zur Verantwortung füreinander
}

Berlin ist die Stadt der Naziverbrecher, der so mühevollen wie hartnäckigen Aufarbeitung der Shoah und gleichwohl der steten Gefährdung der Demokratie von ultrarechts bis ganz links - und das bis heute. Berlin ist aber noch mehr: Neben anderen „Fremden“ leben in dieser Metropole, wo sich die Welt und Startups treffen, inmitten von etwa vier Millionen Deutschen aus Ost und West auch gut 25.000 jüdische Israelis sowie etwa 60.000 Palästinenser. Deren Zusammenleben macht etwas mit ihnen und ihren deutschen Nachbarn. Das vorliegende Buch über dieses „Dreieck“ zwischen Deutschen, Palästinensern und Israelis in Berlin ist eine Momentaufnahme aus dem Sommer 2018. Sie zeigt, dass die Deutschen zwar die Israelis in der Stadt wahrnehmen, aber sie begegnen ihnen vielfach mit antisemitischen Gefühlen. Viele Israelis fühlen sich gleichwohl gut in dieser Stadt. Eine große Zahl von ihnen ist in Berlin, um antipalästinensischen Feindbildern in der Heimat zu entweichen. Andere kommen erst hier mit Palästinensern in Kontakt. Die deutsche Bevölkerung nimmt in der Regel die viel zahlreicheren Palästinenser genauso wenig wahr wie ihre Traumata. Viele Deutsche neigen zunächst einmal dazu, die zum Teil undifferenzierte Unterstützung Israels durch die Regierung zu teilen. Aber im direkten Kontakt zwischen den drei Gruppen ändert sich dann doch vieles, und darum geht es in diesem Buch. Es bildet eine spezielle Berliner Koexistenz ab, die in manchen Momenten wie ein Treibhaus für mehr Frieden im Nahen Osten erscheint.

Dieser Befund macht die vorliegende Sammlung von einhundert Gesprächen von Katharina Galor und Sa'ed Atshan mit Israelis, Palästinensern und Deutschen in Berlin so wichtig wie grundlegend. Ihr Buch ist zwar nur eine erste Studie über die drei aufeinanderstoßenden Nationalitäten, aber diese lässt vielleicht schon einen Schluss zu; und der stammt nicht von dem deutschen Vorwortschreiber, der gewiss gerne etwas Nettes über die deutsche Hauptstadt hören möchte, sondern eben von der Israelin Galor und Atshan aus dem palästinensischen Ramallah. Ihre Gesprächssammlung und Schlussfolgerung legen die Vision nahe, dass an der Spree Ideen dazu reifen, wie eine friedliche Zukunft zwischen Palästinensern und jüdischen Israelis - jenseits des schon einhundert Jahre alten israelischarabischen Konflikts - aussehen könnte. Gewiss, es klingt surreal, wenn die Stadt der schlimmsten Verbrechen im 20. Jahrhundert auf eine so unvermutete Weise zu einem Segensplatz für das 21. Jahrhundert würde. Aber genau davon ist in diesem Buch die Rede. 
Vor Jahrzehnten hätte man sich noch nicht vorstellen können, dass Israelis überhaupt je in Berlin leben würden. Von den etwa 160.000 Berliner Juden vor ihrer Verfolgung, hatten nur etwa 8.000 die Shoah überlebt. Die meisten Neuberliner, die von 1946 an die Gemeinde wieder aufbauten, waren nicht Nachfahren der Gemeindeglieder aus der Vorkriegszeit; sondern das waren Überlebende, die der Krieg nach Berlin verschlagen hatte und die zunächst nicht vorhatten, in Deutschland zu bleiben. Sie stammten oft aus Europas Osten - und blieben. Diese mittlerweile seit zwei oder gar drei Generationen in Berlin lebenden deutschen Juden sind oft orthodox und haben sich zwischen jüdischer Schule, Synagoge und nun auch dem orthodoxen Rabbinerseminar in der Brunnenstraße ein halbwegs religiös-jüdisches Leben aufbauen können.

Die Israelis, die heute in Berlin leben, sind zumeist säkular und kommen aus allen gesellschaftlichen Schichten. In der Regel sind es junge Leute und solche mittleren Alters, die sich nicht von den „Stolpersteinen“ und der allgegenwärtigen Shoah-Erinnerung abschrecken lassen. Viele jüdische Israelis fühlen sich gerade durch dies stete Erinnern und Mahnen in Deutschland willkommen. Es hilft ihnen, die Traumata jener Geschichte zu verarbeiten. Von diesen Israelis erfahren wir viel in der Presse. Sie freuten sich vor einigen Jahren nicht zuletzt darüber, dass Butter und das Milky-Produkt der israelischen Firma Strauss in Berlin billiger waren als in Tel Aviv. Sie besitzen trendige Bars und überraschen mit tabulos erscheinenden, aber von Menschlichkeit geprägten Szenestücken am GorkiTheater.

Und dann gibt es eben in Berlin die viel größere Gruppe von Palästinensern, die sich bisher kaum in den Medien spiegelt; es sei denn, es geht um Mitglieder krimineller Clans. Tatsächlich ist das gesellschaftliche Spektrum dieser Araber aber genauso weit gespannt wie bei den Israelis. Die ersten Palästinenser kamen schon zu DDR-Zeiten als Studenten der Medizin und Ingenieurwissenschaften, später auch als Gastarbeiter nach Berlin; dann folgten Flüchtlinge über Libanon aus den besetzten Gebieten. Seit jüngerem lockt viele einfach nur dieses neue Berlin. Dass sie da neben den „fremden“ Deutschen auch auf die ihnen misslich vertrauten Feinde aus Israel stoßen würden, war gewiss den meisten zunächst nicht klar. Viele fühlen sich auch - im Schatten der jüdischen Israelis - wie übersehen oder als palästinensische Terroristen an Israelis stigmatisiert. Dass ihr Leben in Berlin womöglich darauf zurückzuführen ist, dass ihre Eltern oder Großeltern aus der Heimat fliehen mussten, wollen viele Deutsche nicht wissen.

In Berlin wie in Deutschland spricht man vor allem vom Leid der Juden in der Shoah. Die Nakba aber - eben diese „Katastrophe“ der Flucht und Vertreibung von etwa 700.000 Palästinensern in Israels Gründungskrieg 1948 - kommt im deutschen Denken kaum vor. Es ist unter Deutschen fast ein Tabu, die Traumata von Shoah und Nakba in ihrer historischen Abfolge und so in einer Kausalität zu 
sehen; zwar nicht im Vergleich, wohl aber in der Anerkennung, dass Menschen nun einmal von ihren Traumata geprägt werden, die darum auch zu respektieren sind - ganz egal, welche es sind. Weder Palästinenser noch Israelis und auch nicht die Deutschen in diesem Berlin waren wohl zunächst darauf aus, mit den anderen in Beziehung zu treten.

Katharina Galor und Sa'ed Atshan aber entdecken nun bei ihren Gesprächen mit so manchen Israelis und Palästinensern, wie sie doch den Kontakt suchten und darüber ihre ererbte Feindschaft zunächst in Frage stellten, um sie dann endlich aufzugeben. So begannen diese Gesprächspartner, nach den Worten der Autoren, „ihren Dämonen zu entkommen“. Oberflächlichen deutschen Beobachtern in Berlin mag schon auffallen, wie sich Israelis und Palästinenser in ihrem Lebensstil angleichen: Israelis essen palästinensischen Hummus und halten ihn für israelisch; beide hören die Musik ihrer Region, und sie trinken beide ihren Tee mit Zucker und Nana. Viele Israelis und Palästinenser aber entdecken darüber hinaus tiefe Gemeinsamkeiten, deren Anerkennung der Kriegs- und Terroralltag in der Heimat aber quasi verboten hatte.

Womöglich ist Berlin selber ein Trigger für diesen Wandel. So war es wenigstens bei den Autoren, die nichts nach Deutschland zog. Im Gegenteil: beide verbanden mit Berlin nur Argwohn und Feindschaft. Dabei ist Katharina Galor 1966 in Düsseldorf geboren. Sie stammt aber aus einer österreichisch-ungarisch-jüdischen Familie aus Siebenbürgen, die sich - um dem Kommunismus zu entkommen und nur wegen der vertrauten Sprache - in Deutschland niederließ. Das geschah noch zu einer Zeit, als in israelischen Pässen stand, sie hätten für alle Länder Gültigkeit „... außer Deutschland“. Katharinas Eltern wollten darum auch nur vorübergehend in diesem Land bleiben; schließlich hatten die Nazis den Vater und seine Familie nach Auschwitz deportiert. Bis zum Abitur mit 19 Jahren erlebte Katharina dann ein ihr feindlich gesonnenes Deutschland. Sie sah sich als jüdische Ausländerin und bekam von ihren direkten Nachbarn einen so starken Antisemitismus zu spüren, dass diese Erfahrungen „ihr Leben prägten“.

Dass Katharina Galor nach 30 Jahren in Frankreich, Israel und in den Vereinigten Staaten dennoch nach Deutschland und nun nach Berlin kam, ergab sich daraus, dass sie ihren Mann nicht allein nach Berlin ziehen lassen wollte. Der aus einer deutsch-jüdischen Familie stammende Kulturwissenschaftler Michael Steinberg wurde nämlich 2016 Präsident der American Academy. Diese 1994 gegründete amerikanische Einrichtung fördert den wissenschaftlichen und kulturellen Austausch zwischen den USA und Deutschland. Katharinas Ehemann versetzte so seine zunächst widerwillige Ehefrau in ein Berlin, das sich aber als „anders“ herausstellte, als Katharina es befürchtet hatte. Deutschland hatte sich in ihren Augen insbesondere in Bezug auf den Holocaust „zum Besseren gewandelt“, stellte sie fest. „Bewegend und wie die Zurückgewinnung verloren 
gegangenen Besitzes“ erlebte sie ihren Einzug in die Wohnung in der Charlottenburger Sybelstraße 9. In dem Privathaus hatten die drei Kinder des berühmten Direktors vom „Städtischen Konservatorium für Musik in Berlin“ Gustav Hollaender von 1936 bis 1941 versucht, den geordneten Betrieb aufrecht zu erhalten, nachdem die eigentliche Schule 1935 mit dem Rausschmiss der jüdischen Pädagogen „arisiert“ worden war. Kurt Hollaender, Susanne Landsberg, geb. Hollaender und Melanie Herz-Hollaender betrieben die „Jüdische private Musikschule Hollaender“, aber wurden von dort zwischen 1941 und 1943 deportiert und ermordet. Diese Geschichte fand Katharina ausführlich dargestellt. „Hier wurde nichts verschwiegen. Ich hatte Gänsehaut, als ich diese Geschichte las und dass ich da quasi als jüdische Nacherbin einziehen konnte, berührte mich“, erzählt Katharina. Neue Freunde und viele Gespräche gaben ihr bald das Gefühl, in diesem Berlin willkommen zu sein.

Sa'ed Atshan, Jahrgang 1984, wollte nie „einen Fuß auf deutschen Boden setzen“. Der Schüler einer Quäker-Highschool in Ramallah hatte die deutsch-jüdische Geschichte der NS-Zeit durch die Schullektüre von Elie Wiesel und Anne Frank kennengelernt. Er wusste von den Traumata in israelischen Köpfen und sah sich - zusammen mit seiner Familie - den Folgen dieser Traumata durch die Besatzung im Westjordanland ausgesetzt. Später konnte Sa'ed am Quäker-College in Swarthmore in Pennsylvania gleichwohl tiefere Beziehungen zu Juden und Israelis aufbauen und begriff, wie wichtig es ist, einen jeden und eine jede als menschliches Wesen zu begreifen, unabhängig - zum Beispiel - von der brutalen Besatzung der palästinensischen Bevölkerung durch israelische Soldaten, also unabhängig von Strukturen der Macht und ihrer Ungerechtigkeit, wie er in seinem Nachwort schreibt. Überdies lernte Sa'ed über jüdische Freunde den Antisemitismus von heute kennen. Und das weckte ihn vollends auf: „Als Quäker bin ich der festen Überzeugung, dass Antisemitismus in all seinen Formen benannt werden sollte und dass es auch weiterhin intellektuelle Räume geben sollte, um seine verschiedenen Erscheinungsformen zu historisieren.“ Kein Wunder, dass damit der historische Antisemitismus der Deutschen und die Shoah wieder in Sa'eds Blickfeld geriet. Als ihn Katharina, deren Trauzeuge er bei der Hochzeit mit Steinberg gewesen war, nach Berlin zu kommen beschwor, konnte er der guten Freundin diese Bitte nicht ausschlagen.

Sa'ed reiste also an die Spree: Denn ,wenn Katy [Katharina] ihr Zögern überwinden konnte, in ihr Geburtsland zurückzukehren, nachdem sie und ihre Familie dort allein wegen ihrer jüdischen Identität unaussprechliche Schrecken erlebt hatten, dann sollte auch ich in der Lage sein, eine längere Zeit in Berlin zu verbringen und dieses Forschungsprojekt mit ihr durchzuführen." Schon der türkische Taxifahrer vom Flughafen Tegel in die Stadt habe bei ihm das Gefühl geweckt, dass dieses Berlin lebendig und von sprudelnder Humanität sei, sagt 
Sa'ed. Katharina hatte ihn aber auch nicht nur einfach mal so eingeladen. Sie war in Berlin auf diese seltsame Koexistenz zwischen Deutschen, Palästinensern und Israelis gestoßen und wollte mit Sa'ed Interviews zum komplexen Verhältnis dieser drei Nationen zueinander führen. Dass sich Sa'ed dann in seinen zwei Monaten im Sommer 2018 mit Berlin anfreundete, dass er diese facettenreiche Gesellschaft und Auseinandersetzung sowohl mit dem Antisemitismus als auch mit dem Strom der Flüchtlinge zu schätzen begann, und dass er sich als schwuler Aktivist und Queer-Forscher in Berlin schnell zu Hause zu fühlen begann, soll nur erwähnt werden.

Denn in den Mittelpunkt trat für beide die Umsetzung einer selbst auferlegten moralischen Pflicht. Beiderseits tief, aber gegensätzlich von eigenen Traumata und Erfahrungen gezeichnet, begannen sie, spontan oder mit Fragebogen ihre Mitmenschen in Berlin nach deren Prägungen durch Erfahrung und Vorurteil zu befragen. So entstand diese Interviewsammlung weniger aus einem nüchternen wissenschaftlichen Interesse der Israelin. Katharina ist schließlich nicht Soziologin, sondern Archäologin, die neben anderem ein Standardwerk zur Archäologie von Jerusalems Anfängen bis zu den Osmanen (zusammen mit dem Tübinger Archäologen Hanswulf Bloedhorn) schrieb. Die Berliner ethnographische Feldarbeit entstand auch weniger aus nüchterner Neugier des arabischen Anthropologen Sa'ed, der sich aus seiner heimatlichen Erfahrung heraus bisher vor allem auf Friedens- und Konfliktstudien konzentrierte. Als Quäker befasste er sich auch mit den christlichen Minderheiten sowie der gesellschaftlichen Akzeptanz für Homosexualität in seiner Heimat. Sa'eds jüngstes Buch beschäftigt sich mit „Queer Palestine and the Empire of Critique.“ Die Israelin und der Palästinenser bildeten vielmehr ein Tandem, um aus ihrer persönlichen Übereinkunft heraus die asymmetrischen Beziehungen zwischen den Nationen in Berlin vor dem Hintergrund der offiziellen Positionen der deutschen Regierung und der israelischen zu beleuchten.

Die 50 strukturierten und 50 informellen Gespräche mit Deutschen, Israelis und Palästinensern im Alter von 16 bis 81 wurden auf Deutsch, Hebräisch, Arabisch und Englisch geführt und erbrachten eine Vielfalt von Meinungen und Argumenten, die sich nur schwer zusammenfassen ließen. Wie sollte man zum Beispiel jene potentiellen Gesprächspartner bewerten, die sich allein schon der Idee verweigerten, bei einem israelisch-palästinensischen Projekt mitzuarbeiten? So gab es etwa Gesprächspartner, die berufliche Nachteile befürchteten. Die meisten wollten ihren Namen nicht genannt sehen; damit waren nur sechs einverstanden. Ein Palästinenser wollte zwar Israels Besatzung knallhart kritisieren, zugleich aber keineswegs als Antisemit gelten. In der Regel führten Katharina und Sa'ed die Gespräche gemeinsam; hatte sich doch rasch herausgestellt, dass ein Israeli einer Israelin gegenüber anders spricht als gegenüber dem binationalen 
Tandem. Deutsche und israelische Amtsträger gehörten genauso zu ihren Gesprächspartnern wie Ärzte oder Fitnesstrainer, Friseure und Bauarbeiter.

Es kam den beiden Autoren darauf an, möglichst viele Stimmen einzufangen; dabei konnten sie nicht statistisch sauber verfahren. Wie im Schneeballsystem ging es vom einen zum nächsten. Fast immer begannen die Gespräche mit persönlichen Eindrücken und wechselten dann zu Gedanken über Politik und Werte. Oft war der Ausgangspunkt das Vorverständnis von historischen Vorgängen durch die Eltern. Da kann sich ein Vater auf jüdischer Seite zum Beispiel nicht damit abfinden, dass die Tochter in jenes Berlin ziehen will: „Mein Vater hörte auf, mit mir zu reden“, berichtet die Israelin, die ein Stipendium nach Berlin gelockt hatte. Junge Deutsche seien ja womöglich unschuldig, „aber die Alten, da weiß man nie, was sie getan oder nicht getan haben, “ hatte der Vater gemeint. Der kam dann aber selber an die Spree und lernte das neue Berlin schätzen. So erlebt der Leser mit, wie sich eingespielte Narrative und Vorurteile aufweichen. Er lernt Berliner Einrichtungen kennen, an denen sich Israelis und Palästinenser bemühen, ihre Vorbehalte zu überwinden und zu einer neuen Solidarität zu finden. Sa'ed muss freilich auch miterleben, dass er als Palästinenser, der sich sein Leben lang für den Frieden einsetzt, nicht einfach eine Rede im Jüdischen Museum Berlin halten darf, weil die israelische Regierung gegen den Auftritt des vermeintlichen Feindes einschreitet und sich ein Vetorecht anmaßt, das ihr nicht zusteht. Zwar müssen sie immer wieder mal ihren eigenen Zorn über den Judenhass oder eine Islambeschimpfung bei Interviewten im Zaun halten. Aber auf der „Jagd nach den Dämonen historischer Traumata“ finden sie bei ihren Gesprächspartnern auch viel menschliche Zuwendung.

Es gibt wohl kaum ein schwierigeres Thema als die Aussöhnung zwischen Deutschen und Israelis. Dabei gerät immer wieder aus dem Blick, dass dieser Ausgleich nur gelingen kann, wenn das gesamte Bild gesehen wird, also auch die israelisch-palästinensische Wirklichkeit. Bisher ist es in Deutschland nicht üblich, über die Zustände in den palästinensischen Gebieten zu sprechen, sollte das die Kritik an der Regierung in Jerusalem enthalten. Oft gilt tatsächlich als „,antisemitisch“, wer nur Israels Regierungspolitik kritisiert. Noch immer hält die Politik jene „Vision der Zwei-Staaten-Lösung“ hoch, obwohl sich diese aus Mangel an Land für Palästinenser und wegen der demographischen Entwicklungen auf beiden Seiten kaum mehr umsetzen lässt. Die israelische Demokratie ist aber gefährdet, sollten immer mehr Araber in den israelischen Staat kooptiert werden, ohne dieselben Bürgerrechte $\mathrm{zu}$ bekommen wie jüdische Israelis. Wenn die Stabilität Israels in sicheren Grenzen wirklich historische Verantwortung und deutsche Staatsräson ist, wie Bundeskanzlerin Angela Merkel in ihrer bewegenden Knesset-Rede 2008 sagte, dann schließt das mit ein, die selbstzerstörerischen Züge der jüngsten Regierungen in Jerusalem genauso anzusprechen wie die kor- 
rupte Unfähigkeit der palästinensischen Autonomiebehörde in Ramallah, die mit Millionen Euro am Leben gehalten wird.

In dieser Interviewsammlung wird deutlich, dass Offenheit zwar brüskiert und schmerzt und wohl nicht in das diplomatische Gepäck der Politiker gehört. Aber gesellschaftliche Wortführer, Denker in Kultur, Kirche und Wissenschaft sollten wahrhaftiger miteinander reden, so wie das in diesem Buch geschieht. Diese Offenheit muss jeden Antisemitismus sowie jede Islamfeindlichkeit bekämpfen und sie sollte nicht nur Respekt vor nationalen Identitäten mit ihren Traumata haben, sondern - wie die Feldforscher Sa'ed und Katharina - um Heilung bemüht sein, damit wir über die gegenseitige Zuwendung zu einer besseren Zukunft kommen. In Berlin scheint es dafür Ansätze zu geben, die in diesem Buch nachzulesen sind.

Berlin, Februar 2021

Jörg Bremer 